\title{
Perioperative Search for Circulating Tumor Cells in Patients Undergoing Radical Cystectomy for Bladder Cancer
}

\author{
A. Karl ${ }^{1}$, S. Tritschler ${ }^{1}$, S. Hofmann², C. G. Stief ${ }^{1}$, C. Schindlbeck ${ }^{2}$ \\ ${ }^{1}$ Department of Urology, Ludwig-Maximilians-University, LMU, Munich, Germany, \\ ${ }^{2}$ Department of Obstetrics and Gynecology, Ludwig-Maximilians-University, LMU, Munich, Germany
}

\begin{abstract}
Objective: Despite having an organ confined tumor stage at the time of radical cystectomy, a certain number of bladder cancer patients will develop local or distant metastases over time. Currently there are no reliable serum markers for monitoring and evaluating risk profiles of urothelial cancers. Several studies suggest that detection of Circulating Tumor Cells (CTC) may correlate with disease status and prognosis at baseline and early in the treatment of cancers. The presence of CTCs in whole blood before and during radical cystectomy could provide further information on disease status, and could be used as an indicator to determine the need for adjuvant or even perioperative
\end{abstract} chemotherapy.

Methods: From 03/2009 to 05/2009, five patients with histologically proven transitional cell carcinoma of the urinary bladder participated in this study. All patients were admitted to the hospital for radical cystectomy $(\mathrm{rCx})$. A standard or extended lymph node dissection was performed in all cases. Preoperative CT or MRI scans revealed no distant or local metastases. Median age was 66.8 years (55-81 yrs). After obtaining informed consent from each patient, approximately 30 $\mathrm{mL}$ of peripheral blood was taken immediately before $\mathrm{rCx}$ and again during surgical removal of the urinary bladder from the patients' body. As additional parameters, operation time (OR) for surgical removal of the bladder and the amount of blood volume that was used for the detection of CTCs were recorded. Obtained blood samples were processed using the CellSearch System (Veridex $\left.{ }^{(}\right)$within 48 hours of collection. CTCs were identified and quantitated using the Cell-Search System, followed by re-evaluation of the provided results by specially trained and experienced personal. (CS, SH)

Results: CTCs were detected before and during surgical removal of the urinary bladder in one of five patients $(20 \%)$. In the one patient positive for CTC, two CTCs were detected in the blood sample that was obtained before surgery (analyzed blood volume was 25 $\mathrm{mL}$ ). There was one CTC detected in the blood sample that was obtained during surgical removal of the urinary bladder (analyzed blood volume was 27 $\mathrm{mL}$ ). There was no rise in the amount of CTCs during surgical procedure. The final pathological report of this patient showed an advanced tumor stage (T3b, N0, R1). In the other patients, no CTCs were detected at all, neither before rCX nor right after surgical re- moval of the bladder. Pathological stage for these patients ranged from pT1m G3 - p'T2b G3. None of these patients showed lymph node involvement. An average of 14.6 lymph nodes (5-40 LNs) were obtained. OR time to surgical removal of the urinary bladder ranged from 60 minutes to 150 minutes (mean 82 min.).

Conclusions: Although only a very small group of patients was analyzed in this study, the presence of CTCs seems to be correlated with an advanced tumor stage. Therefore the detection of CTCs could be used for an optimized assessment of a patient's disease status in urothelial cancer. A further aim of this study was to assess whether surgical manipulation during radical cystectomy is associated with a release of CTCs into the vascular system. None of the patients who were negative for CTCs before surgery showed CTCs during surgical removal of the bladder, suggesting that there was no release of CTCs during surgery. However, further study is needed to prove these findings and evaluate the significance of CTCs as an indicator for therapeutic decisions.

Key words: bladder cancer, circulating tumor cells, CTC, cell search system, perioperative tumor cell detection

\section{INTRODUCTION}

Carcinoma of the urinary bladder is the fifth most frequent malignant disease in the Western World. In the US approximately 70,980 new cases of bladder cancer will be diagnosed and 14,330 people will die from this disease in 2009 [1]. Around 75\% of patients are diagnosed initially with non-muscle-invasive tumors that can be treated locally with transurethral tumor resection, but $50-70 \%$ of these patients show recurrence within the first 2 years after initial diagnosis [2].

Generally, in most countries radical cystectomy $(\mathrm{rCx})$ represents the gold standard treatment for muscle-invasive bladder cancer. However approximately one third of patients diagnosed with muscle-invasive bladder cancer have undetected metastases at the time of treatment of the primary tumor [3], and 25\% of patients treated by radical cystectomy present with lymph node involvement at the time of surgery [4].

In addition, approximately $50 \%$ of patients with muscle-invasive urothelial cancer develop metastases within 2 years of operation and subsequently die of the disease [5]. 
Since there are patients who develop metastases over time despite an organ confined primary tumor stage, there is a definite need for new diagnostic tools that more accurately reflect the patient's actual disease status. Identifying those patients who are more susceptible to development of metastases over time could lead to optimization of therapeutic management.

In this study we hypothesized that the presence of CTCs before or even during radical $\mathrm{rCx}$ (due to surgical manipulation) could give further insight into the patient's actual disease status. The presence of CTCs could potentially be an indicator of eventual tumor behavior. Based on the detection of CTCs during $\mathrm{rCx}$ a more aggressive individualized therapy regimen could be initiated which could lead to an improvement in clinical outcome. As surgical manipulation and manual compression of the tumorous area could be associated with the release of tumor cells into the vascular system, this study aimed to measure whether CTCs could be detected during the surgical removal of the urinary bladder.

To our knowledge this is the first study that aims to detect CTCs in patients with urothelial cell carcinoma during the performance of radical cystectomy.

\section{Material AND Methods}

In this pilot study we included five patients with previously histologically proven transitional cell carcinoma of the urinary bladder. All patients warranted radical cystectomy due to their tumor stages. Preoperative tumor stages ranged from a multifocal T1 G3 tumor to T2 G3 tumors. All patients underwent radical cystectomy at our institution sometime between 03/2009 and $05 / 2009$. Two men and three women were included, median age was 66.8 years (55-81 yrs). After informed consent, approximately $30 \mathrm{~mL}$ of peripheral blood was obtained using three Cell Safe Preservation Tubes (Immunicon, Huntingdon Valley, PA ${ }^{\circledR}$ ) before the performance of radical cystectomy and again during the surgical removal of the urinary bladder from the patient's body. The time from the beginning of the operation to the actual surgical removal of the urinary bladder was recorded for each patient as an indirect indicator for the intensity of surgical manipulation. The exact blood volume used for detection of CTCs was recorded for each patient. A standard or extended lymph node dissection was performed. All patients were preoperatively staged (CT/MRI scan) by an experienced radiologist as negative for local or distant metastases.

\section{ISOLATION AND ENUMERATION OF CTCS}

Blood samples were maintained at room temperature and processed using the CellSearch System within 48 hours of collection [6]. The Cell Search System (Immunicon(C), Huntingdon Valley, PA) was used for isolation and enumeration of CTCs. This system works semi-automatically to prepare the sample. The procedure enriches the sample for cells expressing EpCAM using antibody-coated magnetic beads and labels these cells with a fluorescent nucleic acid dye 4',6-diamidino-2-phenylindole (DAPI). Fluorescentlabeled monoclonal antibodies specific for leukocytes (CD45-allophycocyan) and epithelial cells (cytokeratin 8,18,19-phycoerythrin) are used to distinguish epithelial cells from leukocytes. The identification and quantification of circulating tumor cells was performed by a semi-automated fluorescence based microscopy system that enables computer generated reconstruction of cellular images. Afterwards, the hits that were generated by the system were reevaluated by specially trained and experienced personal (CS,SH).

\section{CTC DEFINITION}

To qualify as a CTC, an object must be round or oval, have a nucleus (as determined by positive 4',6-diamidino-2-phenylindole staining) contained within the cytoplasm (as determined by positive cytokeratin 8,18,19 -phycoerythrin staining), and lack expression of CD45 (as determined by negative CD45-allophycocyanin staining).

\section{RESULTS}

Using the Cell-Search System $\left(\right.$ Veridex $^{\complement}$ ), CTCs were detected before and during surgical removal of the urinary bladder in one of five patients (20\%). In the one patient positive for CTCs, two CTCs were detected in the sample obtained before $\mathrm{rCx}$ (analyzed sample volume of $25 \mathrm{~mL}$ ). In the blood sample obtained during surgical removal of the urinary bladder only one CTC was detected in a sample volume of $27 \mathrm{~mL}$. The final pathological report for this patient showed an advanced tumor stage (T3b, N0, R1). However, no rise in the number of CTCs was observed during $\mathrm{rCx}$.

No CTCs were detected in the 4 other patients included in the study, neither before rCX nor right after surgical removal of the bladder. Pathological stage for these patients ranged from pT1m G3 - p'T2b G3. None of these patients showed lymph node involvement (patient details see Table 1). On average, 14.6

Table 1. Patient details and final pathological results.

\begin{tabular}{ccccccccc}
\hline Pt Nr & Gender & Age & $\begin{array}{c}\text { Tumor } \\
\text { stage }\end{array}$ & Grading & $\begin{array}{c}\text { LN } \\
\text { status }\end{array}$ & $\begin{array}{c}\text { CTC pre } \\
\text { OP }\end{array}$ & $\begin{array}{c}\text { CTC during } \\
\text { OP }\end{array}$ & $\begin{array}{c}\text { OR time (min) to surg. } \\
\text { removal of the bladder }\end{array}$ \\
\hline 1 & $\mathrm{~m}$ & 58 & pT3b & G3-4 & 0 & 2 & 1 & 150 \\
2 & $\mathrm{w}$ & 69 & pT1G3m & G3 & 0 & 0 & 0 & 70 \\
3 & w & 82 & pT2b & G3 & 0 & 0 & 0 & 60 \\
4 & w & 70 & pT2a & G3 & 0 & 0 & 0 & 70 \\
5 & m & 55 & pT2a, Cis & G3 & 0 & 0 & 0 & 70 \\
\hline
\end{tabular}


lymph nodes (5-40LNs) were obtained. OR time to surgical removal of the urinary bladder was measured as an indirect correlate for surgical manipulation. OR time to bladder removal ranged from 60 minutes to 150 minutes (mean 82 minutes).

\section{DISCUSSION}

In most countries, radical cystectomy generally represents the gold standard treatment for muscle-invasive bladder cancer. Based on older trial results, a Cochrane systematic review [7] suggested an overall survival benefit with radical surgery versus radiotherapy in patients with muscle-invasive bladder cancer. However, despite advances in therapy and the institution of multi-modality therapy, survival rates for bladder cancer, particularly for muscle invasive and advanced disease, have not improved over the last two decades.

Five-year survival rates of $36-54 \%$ have been reported in cystectomy series from major academic centers [8-11]. Chemotherapy has been used both as an adjuvant to surgery and radiation, in a neoadjuvant manner before surgery or along with radiation therapy. Several trials show benefits for adjuvant chemotherapy. However, the results are controversial because of the small sample sizes and confusing analyses and trial designs [12].

Circulating tumor cells have been documented in the circulation of cancer patients since 1869 and discussed in more than 1500 publications. Attempts have been made to detect cancer cells in the blood, bone marrow, or lymph nodes using present PCR techniques for various malignant tumors. However, identification of cancer cell DNA by PCR is laborious, requires very specialized techniques and is not consistently reproducible. Using PCR may be ultrasensitive and not always specific, leading to identification of abnormal DNA that may not necessarily be associated with the cancer cell in question. Identification of CTCs is an alternative to PCR, allowing direct detection of CTCs using immunological technology. This technique seems to be easier, more reliable, and reasonable in terms of cost because it can be automated.

Studies on the detection of CTCs have been performed for different malignancies such as breast, colon, prostate and several others [13-15]. CTCs may even represent an earlier and more reproducible indication of disease status than current imaging modalities as shown by Budd et al. in a study on metastatic breast cancer [16]. In prostate cancer, higher number of CTCs was shown to be associated with poorer outcomes [17]. The presence and number of CTCs seems to correlate with chemotherapy response much earlier than standard radiographic studies; benefit in terms of long-term survival for prostate cancer patients is seen when the CTC count decreases in response to chemotherapy just 2-5 weeks after starting chemotherapy [18].

The CTC detection system was used in this study represents a semi automated technique using immunomagnetic capture to detect CTCs. The basis for the detection of urothelial cancer cells in peripheral blood is that carcinoma cells differ from leukocytes in their gene expression, and each of these cell populations has tissue-specific antigens on the cell surface. The Cell-Search System (Immunicon, Huntingdon Valley, PA $\left.{ }^{(}\right)$combines immunomagnetic enrichment with multiparameter flow cytometric and immunocytochemical analysis. Use of the Cell-Search System for prediction of survival in metastatic breast cancer was approved by the US Food and Drug Administration (FDA) in 2004. The system uses magnetically-labeled anti-EpCAM microbeads and immunocytochemical staining for cytokeratin and CD45. Among epithelial neoplasms, urothelial carcinoma is known to express relatively high levels of EpCAM. An advantage of this detection technique is that it is reproducible across different laboratories and can identify CTCs in different cancer types [19].

Naoe et al. [20] first demonstrated that urothelial cancer cells can be distinguished from leukocytes by using EpCAM and CD45 antibodies through flow cytometric analysis. They found that it is possible to identify bladder cancer cells among peripheral blood mononuclear cells. They demonstrated that the sensitivity for detection of urothelial cancer cells with CellSearch System using single regression analysis of the spiked number of cells vs. the recovered number of cells yielded a good correlation. Urothelial cancer cells were detected in 8 of fourteen patients $(57.1 \%)$ with distant metastasis. These data show that Cell-Search System enables the reliable detection of CTC in blood and seems suitable for assessment of urothelial cancer patients. Also, Gallagher et al. [21] recently reported on the successful detection of CTCs in patients with TCC using the Cell-Search System. They showed that CTCs were detected by antibody capture technology in $44 \%$ of patients with metastatic TCC. Higher numbers of CTCs were seen in patients with a greater number of metastatic sites. One-third of patients had five or more CTCs, providing a potential early marker to monitor response to chemotherapy.

To our knowledge this is the first study aiming to detect CTCs during performance of radical cystectomy. All patients were staged initially to be negative for local or distant metastases using CT/MRI scanning. One patient revealed an advanced tumor stage (T3b, N0, R1) in the final pathological report. This patient turned out to be the one in whom CTCs were detected using the Cell-Search System before and during $\mathrm{rCx}$, suggesting that CTCs could be used as a sensitive tool for the assessment of actual disease status.

Current literature reports demonstrating that CTCs were found only in patients with advanced and metastatic disease were confirmed in our study, as all patients negative for CTCs before radical cystectomy had histologically proven organ confined tumor stages.

Our hypothesis that surgical manipulation during $\mathrm{rCx}$ could be associated with a release of CTCs into the vascular system was not supported by the obtained results. No CTCs were detected at the time of surgical removal of the bladder from the patient's body in patients with localized disease. Also in the one patient positive for CTCs before the operation, no rise in CTCs at the time of surgical removal of the urinary bladder was detected. Therefore we assume that surgical manipulation did not lead to an additional release of tumor cells into the vascular system. It seems un- 
likely that the surgical procedure itself would lead to a release of tumor cells into the vascular system that could be responsible for later development of metastases. To indirectly evaluate the intensity of surgical manipulation of the tumor area during the operation, we recorded the time taken to complete removal of the urinary bladder from the patient's body. The time to surgical removal ranged from 60 to 150 minutes and was not associated with the presence of circulating tumor cells.

As this is a very small study cohort we can not exclude the possibility of tumor cell release into the vascular system during $\mathrm{rCx}$, however from this dataset it seems to be unlikely. Further prospective studies with higher patient numbers would be needed to confirm our conclusions. Nevertheless CTCs could be used as a tool for improved assessment of a patient's disease status and could become the basis for optimization and individualization of therapeutic decision making in the future.

\section{CONCLUSIONS}

Although only a very small group of patients was analyzed in this study, the presence of CTCs seems to be correlated with advanced tumor stage in patients with TCC. Therefore CTCs could be used as a tool for improved assessment of a patient's disease status. A further aim of this study was to assess whether surgical manipulation during radical cystectomy is associated with a release of CTCs into the vascular system. None of the patients who were negative for CTCs before surgery showed CTCs right after surgical removal of the bladder, suggesting that there was no release of CTCs during surgery. However further study is needed to prove these findings and evaluate the significance of CTCs as an indicator for therapeutic decision making.

\section{REFERENCES}

1. Jemal A, Siegel R, Ward E, et al. Cancer statistics, 2009. CA Cancer J Clin. 2009;59(4):225-49.

2. Vaidya A, Soloway MS, Hawke C, et al. De novo muscle invasive bladder cancer: is there a change in trend? J Urol. 2001;165(1):47-50.

3. Prout GR, Jr., Griffin PP, Shipley WU. Bladder carcinoma as a systemic disease. Cancer. 1979;43(6):2532-39.

4. Karl A, Carroll PR, Gschwend JE, et al. The Impact of Lymphadenectomy and Lymph Node Metastasis on the Outcomes of Radical Cystectomy for Bladder Cancer. Eur Urol. 2009;55(4):826-35

5. Messing EM CW. Urothelial tumors of the urinarytract. In: Walsh PC, Retik AB, Vaughan ED Jr, Wein AJ (eds). New York, WB Saunders;1997: pp2327-2410.

6. Schindlbeck C, Stellwagen J, Jeschke U, et al. Immunomagnetic enrichment of disseminated tumor cells in bone marrow and blood of breast cancer patients by the Thomsen-Friedenreich-Antigen. Clin Exp Metastasis. 2008; 25(3): 233-40.

7. Shelley MD, Barber J, Wilt T, et al. Surgery versus radiotherapy for muscle invasive bladder cancer. Cochrane. Database. Syst. Rev. 2002;(1)CD002079.
8. Bassi P, Ferrante GD, Piazza N, et al. Prognostic factors of outcome after radical cystectomy for bladder cancer: a retrospective study of a homogeneous patient cohort. J Urol. 1999;161(5):1494-97.

9. Dalbagni G, Genega E, Hashibe M, et al. Cystectomy for bladder cancer: a contemporary series. J Urol. 2001; 165(4): 1111-16.

10. Ghoneim MA, el-Mekresh MM, el-Baz MA, et al. Radical cystectomy for carcinoma of the bladder: critical evaluation of the results in 1,026 cases. J Urol. 1997;158(2):39399.

11. Stein JP, Lieskovsky G, Cote R, et al. Radical cystectomy in the treatment of invasive bladder cancer: long-term results in 1,054 patients. J Clin Oncol. 2001;19(3):666-75.

12. Sylvester R, Sternberg C. The role of adjuvant combination chemotherapy after cystectomy in locally advanced bladder cancer: what we do not know and why. Ann.Oncol. 2000;11(7)851-56.

13. Cristofanilli M, Budd GT, Ellis MJ, et al. Circulating tumor cells, disease progression, and survival in metastatic breast cancer. N Engl J Med. 2004;351(8):781-91.

14. Sastre J, Maestro ML, Puente J, et al. Circulating tumor cells in colorectal cancer: correlation with clinical and pathological variables. Ann Oncol. 2008;19(5):935-38.

15. Shaffer DR, Leversha MA, Danila DC, et al. Circulating tumor cell analysis in patients with progressive castrationresistant prostate cancer. Clin Cancer Res. 2007;13(7): 2023-29.

16. Budd GT, Cristofanilli M, Ellis MJ, et al. Circulating tumor cells versus imaging--predicting overall survival in metastatic breast cancer. Clin Cancer Res. 2006;12(21): 6403-09.

17. Danila DC, Heller G, Gignac GA, et al. Circulating tumor cell number and prognosis in progressive castrationresistant prostate cancer. Clin Cancer Res. 2007;13(23): 7053-58.

18. Moreno J DJSD. Multi-center study evaluating circulatingtumor cells (CTCs) as a surrogate for survival in men treated for castrationrefractory prostate cancer (CRPC). J Clin Oncol. 2007; 25: 5016.

19. Allard WJ, Matera J, Miller MC, et al. Tumor cells circulate in the peripheral blood of all major carcinomas but not in healthy subjects or patients with nonmalignant diseases. Clin Cancer Res. 2004;10(20):6897-6904.

20. Naoe M, Ogawa Y, Morita J, et al. Detection of circulating urothelial cancer cells in the blood using the Cell Search System. Cancer. 2007;109(7):1439-45.

21. Gallagher DJ, Milowsky MI, Ishill N, et al. Detection of circulating tumor cells in patients with urothelial cancer. Ann Oncol. 2009;20(2):305-08.

Received: August 31, 2009 / Accepted: September 14, 2009

Address for correspondence:

Alexander Karl, MD

Department of Urology

Ludwig-Maximilians-University, LMU Munich

Marchionistr. 15

81377 Munich

Germany

Tel.: $\quad+4989 / 7095-0$

Fax.: +49 89/7095-8890

E-mail: alexander.karl@med.uni-muenchen.de 